\title{
Models of Reflection Nebulae
}

\author{
T. P. Roark and J. Mayo Greenberg \\ Rensselaer Polytechnic Institute \\ Troy, New York
}

\begin{abstract}
A FEASIBILITY STUDY For an OBSERVATIONAL PROGRaM was made to A investigate models of reflection nebulae. Specifically, for a relatively simple model of an interstellar dust cloud with a source of illumination nearby, predictions of the color of the nebula, relative to the source color, at points along any radial direction from the source are sought. Furthermore, from these color differences, the possibility is studied of discriminating between the effects produced by the cloud-sourceobserver geometry and those due to the assumed optical properties of the scattering particles. If such discrimination is feasible, the types of observations that would be most useful in separating the effects must be known to the investigator.

Plane-parallel slab models similar to those of reference 1 in which the scattering particles are distributed isotropically and homogeneously were used. The scattering properties of the particles were assumed to be those obtained by using the Mie theory of electromagnetic scattering.

Single scattering by an ensemble of particles was assumed; a correction factor was applied to allow for the fact that the incident source-light and the light scattered in the nebula are both extinguished exponentially in passing through the nebula. Equation (1) gives the intensity of monochromatic light scattered in a direction $\phi$ regardless of the overall geometrical situation.
\end{abstract}

$$
\begin{aligned}
I_{n}(\phi, \lambda)=I_{8}(\lambda) & \frac{r^{2}}{4 k^{2}} \sec \alpha \mathrm{d} \omega \\
& \times \int_{0}^{T} \int_{a_{1}}^{a_{2}} \frac{1}{R^{2}} F(\Theta, \lambda, a, n) n(a) \mathrm{d} a \mathrm{~d} z \mathrm{e}^{-\kappa(\lambda)\left[L_{r}(z)+L_{n}(z)\right]}
\end{aligned}
$$

where

$$
\begin{array}{ll}
I_{n} & \text { nebular intensity } \\
\phi & \text { viewing angle (see fig. 1) }
\end{array}
$$


$I_{s} \quad$ source intensity in a vacuum

$\kappa$ wave number, $2 \pi / \lambda$

$r$ radius of the source

$\mathrm{d} \omega$ solid angle subtended by viewing device

$\boldsymbol{R}$ distance from source to scattering volume at point $\mathrm{B}$

$F \quad$ scattering amplitude

$\Theta$ scattering angle, $180-\theta$

$a$ radius of scattering particle

$n$ index of refraction of scattering particle

The exponential term on the right-hand side of equation (1) is the single scattering modification term; $\kappa(\lambda)$ is a linear absorption coefficient; $L_{s}(z)$ is the distance $\overline{A B}$ in figure 1 ; and $L_{n}(z)$ is the distance $\overline{B P}$.

The function $n(a)$ is a distribution function for the scattering particle radii. Two forms are assumed for $n(a)$; one yields scattered intensities from a single size particle, and the other is an exponential distribution given by (see ref. 2)

$$
n(a)=n_{0} \exp \left[-5\left(\frac{a}{a_{0}}\right)^{3}\right]
$$

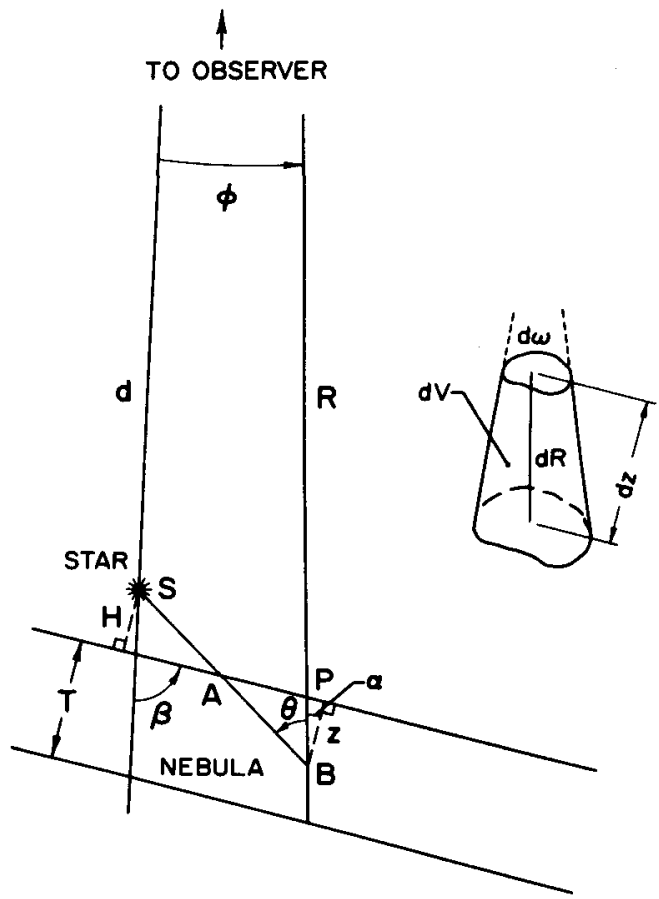

Figure 1. - Basic geometrical configuration for star in front of plane-parallel nebula. Sketch on the right shows detail of scattering volume $\mathrm{d} V$ at $B$. 
For a value $a_{0}=0.50 \mu$, the distribution represented by equation (2) closely parallels that determined in reference 3 .

Ratios of the nebular intensities $I_{n}(\lambda)$ at different wavelengths may be taken and a nebular color obtained. The $B-V$ color difference between the star and nebula is then defined as

$$
\text { Color difference }=2.5 \log \left(\overline{\frac{I_{s}(\lambda)_{V}}{I_{s}(\lambda)_{B}}}\right)-2.5 \log \left(\overline{\frac{I_{n}(\lambda)_{V}}{I_{n}(\lambda)_{B}}}\right)
$$

The integrated light in each band is defined by

$$
I(\lambda)_{i}=\int I(\lambda) Q_{i}(\lambda) \mathrm{d} \lambda
$$

where $Q_{i}(\lambda)$ is the filter-detector transmissivity function for the color " $i$ " ( $i=U, B, V)$. In equation (3), the subscript $s$ refers to the source and $n$, to the nebula. It should be noted that when the source is inside or behind the model nebula an exponential attenuation is introduced in the expression for $I_{s}(\lambda)$. Thus the source colors are those as seen by an observer and are intrinsic source colors only when the source is in front of the nebula. A positive color difference indicates that the nebula is bluer than the source of illumination.

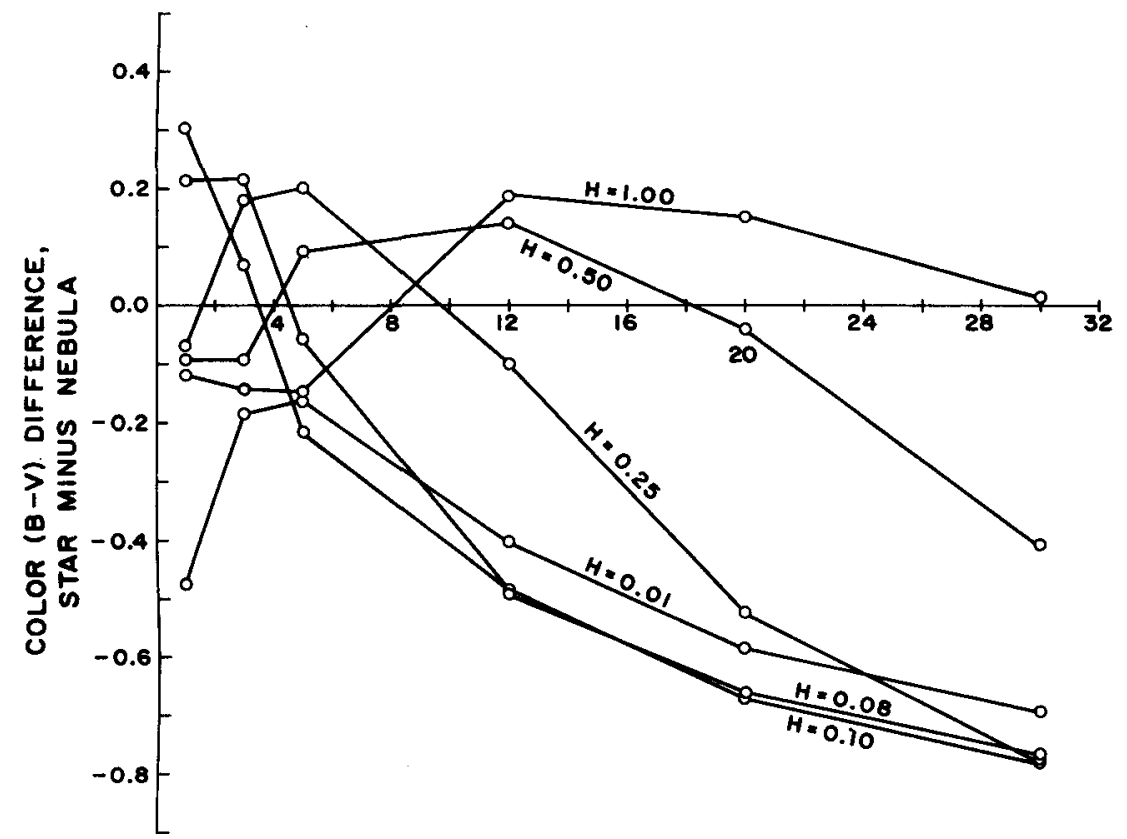

RADIAL DISTANCE, min

FIGURE 2.-Dependence of color difference on changes in distance $H$. Star in front; dielectric spheres: $a_{0}=0.50 \mu ; T=1.0 \mathrm{pc} ; d=160 \mathrm{pcs} ; \beta=90^{\circ} ; \kappa(\lambda)_{V}=1.2$ magnitudes $/ \mathrm{pc}$. 


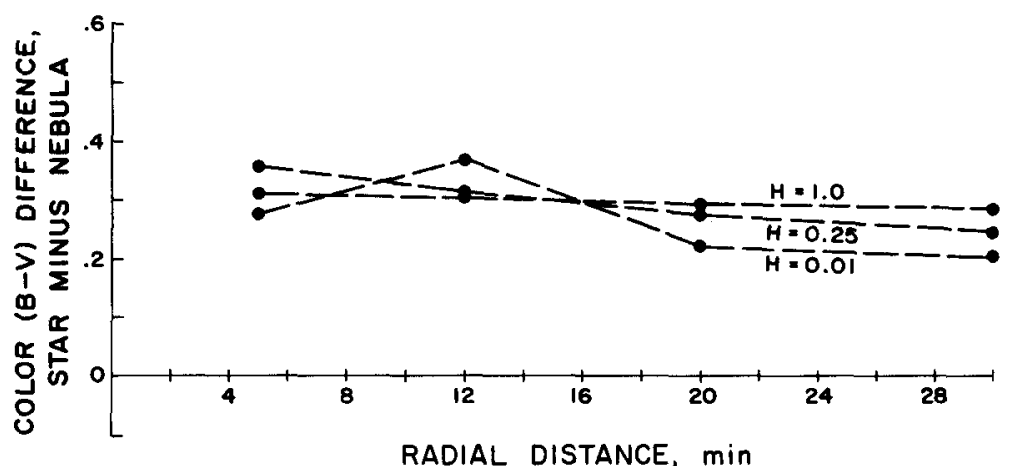

FIGURE 3. - Dependence of color difference on changes in distance $H$. Star in front; graphite spheres: single size, $a=0.05 \mu ; T=1.0 \mathrm{pc} ; d=160 \mathrm{pcs} ; \beta=90^{\circ} ; \kappa(\lambda)_{V}=1.2 ;$ magnitudes $/$ pc.

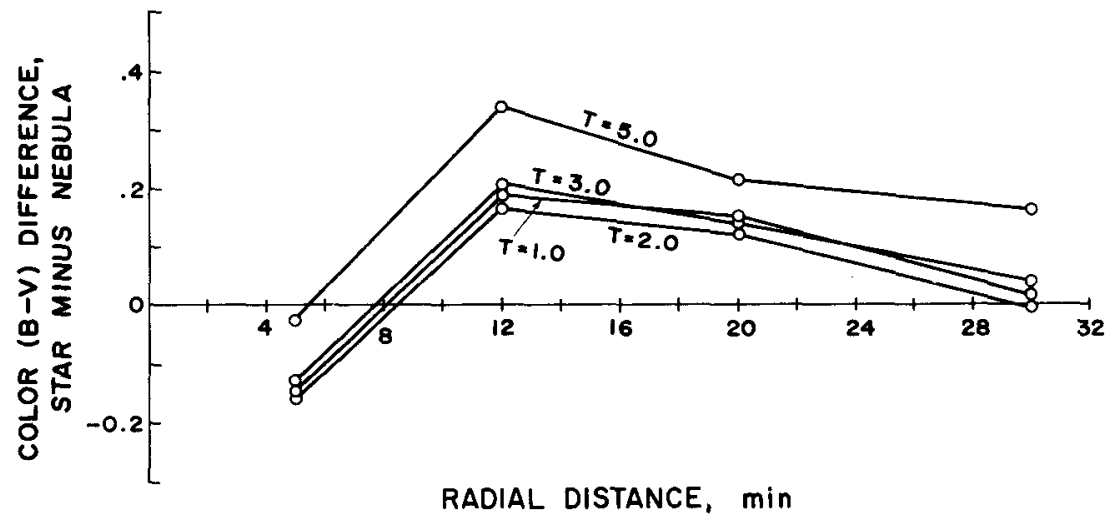

FIGURE 4.-Dependence of color differences on changes in model thickness $T$. Star in front; dielectric spheres: $a_{0}=0.50 \mu ; H=1.0 \mathrm{pc} ; d=160 \mathrm{pcs} ; \beta=90^{\circ} ; \kappa(\lambda)_{V}=1.2$ magnitudes/pc.

Figures 2 to 10 are representative samples of the results. Three types of spherical scattering particles have been used and all three give satisfactory theoretical fits, in the visible region, to the normalized interstellar reddening curve of reference 4 . First are dielectric spheres with a real part to the refractive index of 1.30 , independent of wavelength, and no imaginary part. Their radii are assumed to be distributed according to equation (2) with $a_{0}=0.50 \mu$. Graphite spheres which all have a radius of $0.05 \mu$ are considered next. The refractive indices of reference 5 were used. Schalèn's refractive indices for iron, as quoted in reference 6, were employed. The iron particles were considered because of their historical interest and for comparison with the graphite predictions. 


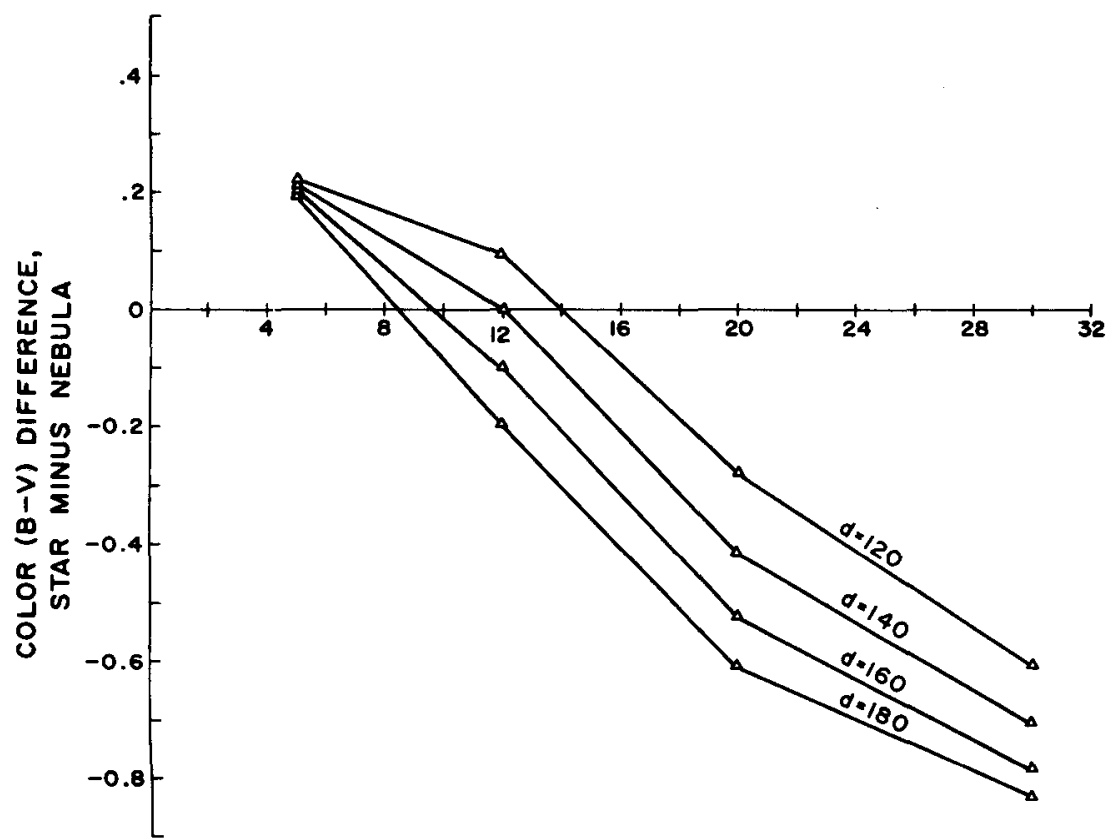

RADIAL DISTANCE, min

FIGURE 5.-Dependence of color differences on changes in observer-nebula distance $d$. Star in front; dielectric spheres: $a_{0}=0.50 \mu ; T=1.0 \mathrm{pc} ; H=0.25 \mathrm{pc} ; \beta=90^{\circ} ; \kappa(\lambda)_{V}=1.2$ magnitudes/pc.

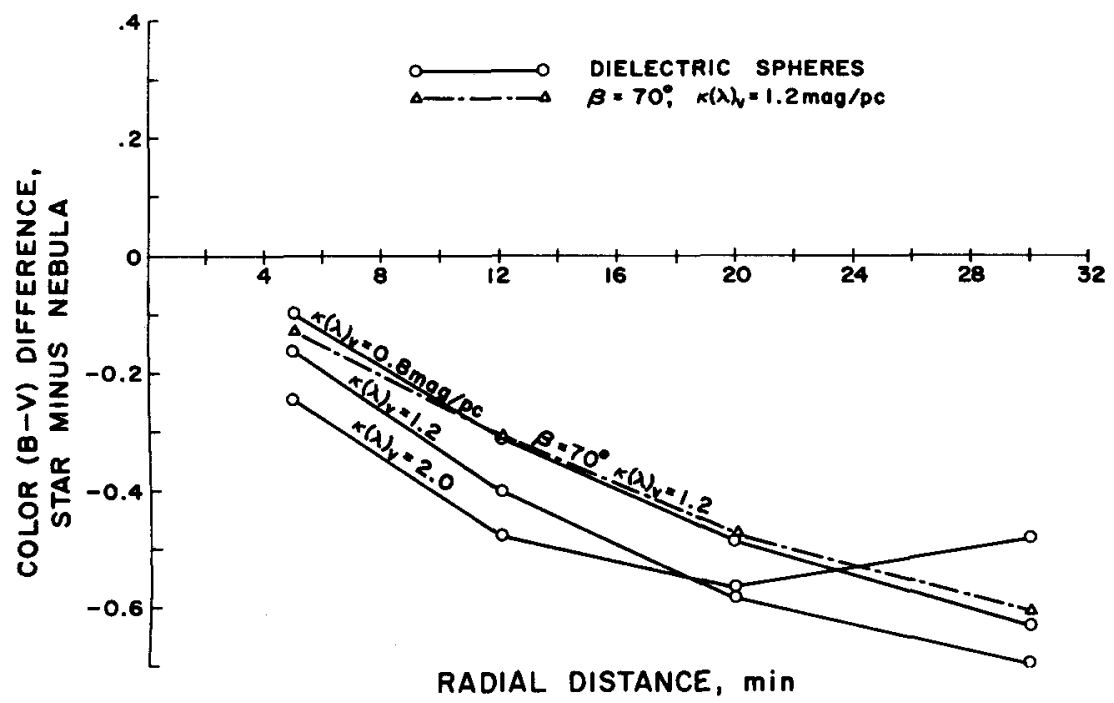

Figure 6. - Dependence of color differences on changes in linear absorption coefficient $\kappa(\lambda)$ and tilt angle $\beta$. Dielectric spheres: $a_{0}=0.50 \mu ; T=1.0 \mathrm{pc} ; H=0.01 \mathrm{pc} ; d=160 \mathrm{pcs}$; solid curves: $\beta=90^{\circ}$. 


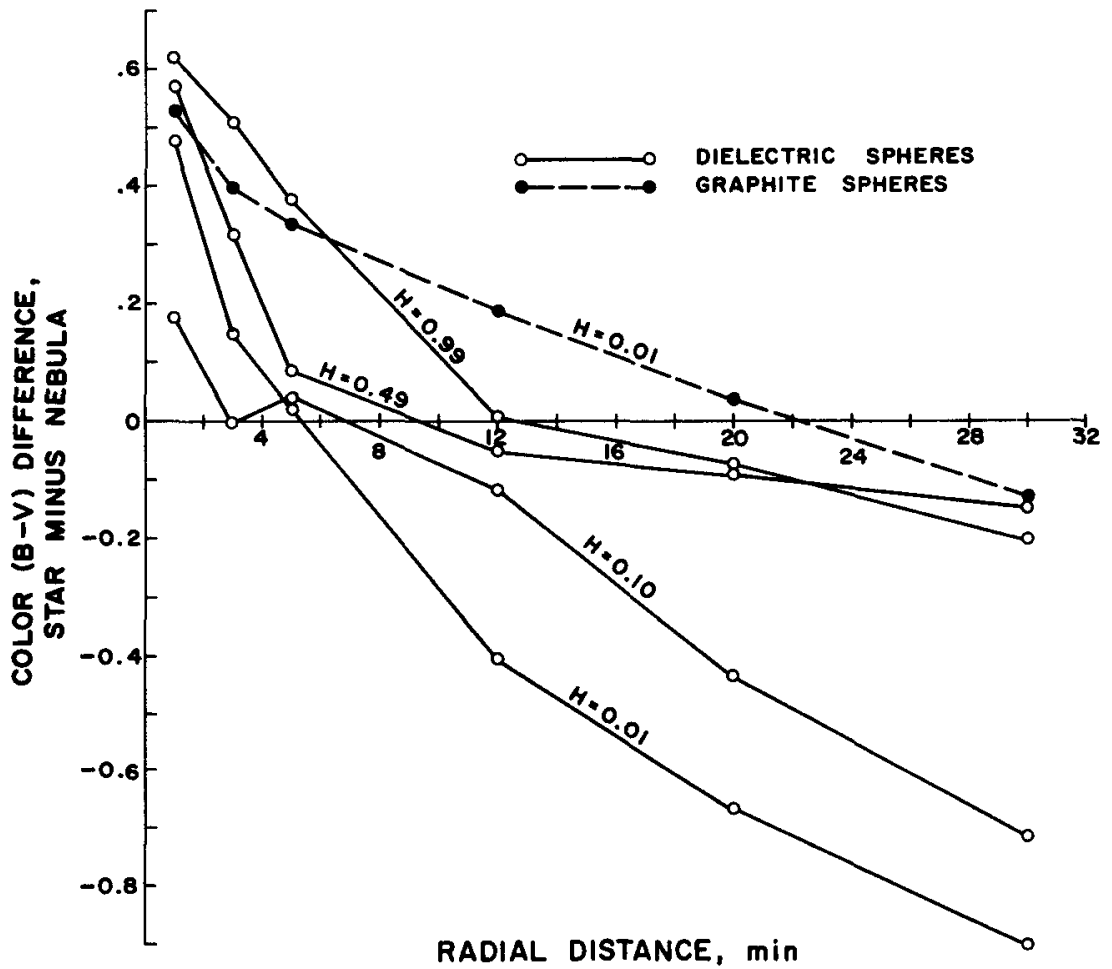

FIGURE 7.-Dependence of color differences on changes in distance $H$. Star inside; $a_{0}$ (dielectric spheres) $=0.50 \mu ; a$ (single size graphite spheres) $=0.05 \mu ; T=1.0 \mathrm{pc}$; $d=160 \mathrm{pcs} ; \beta=90^{\circ} ; \kappa(\lambda)_{V}=1.2$ magnitudes $/ \mathrm{pc}$.

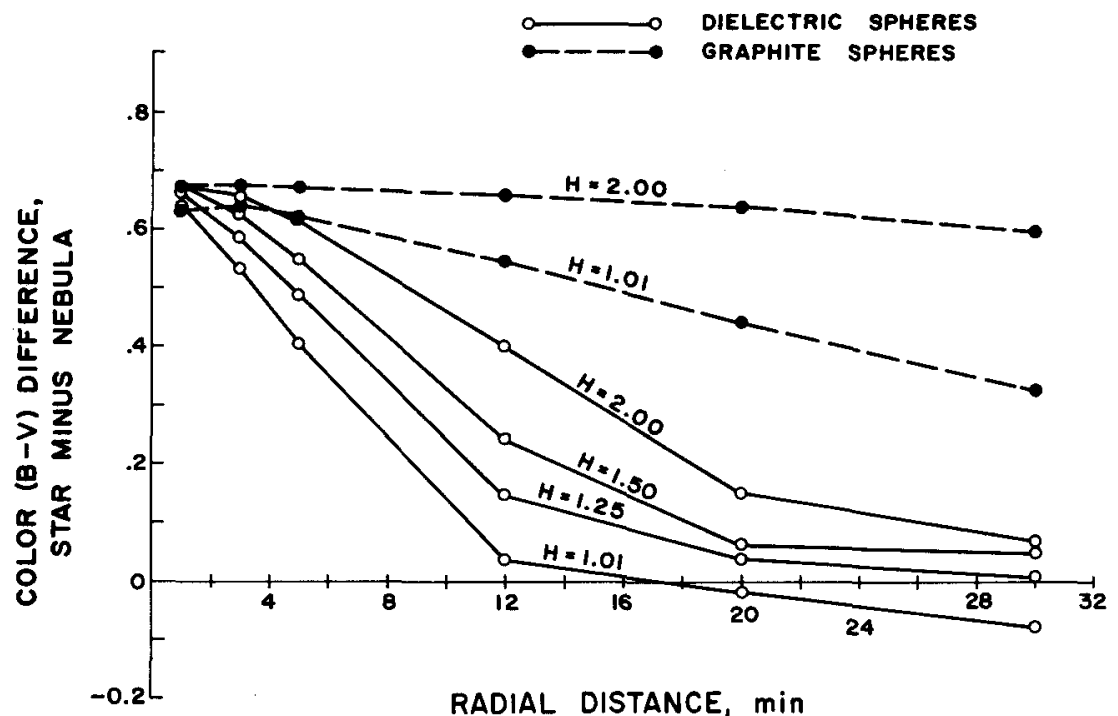

Figure 8.-Dependence of color difference on changes in distance $H$. Star behind: $a_{0}$ (dielectric spheres) $=0.50 \mu ; a$ (single size graphite spheres) $=0.05 \mu ; T=1.0 \mathrm{pc}$; $d=160 \mathrm{pcs} ; \beta=90^{\circ} ; \kappa(\lambda)_{V}=1.2$ magnitudes $/ \mathrm{pc}$. 


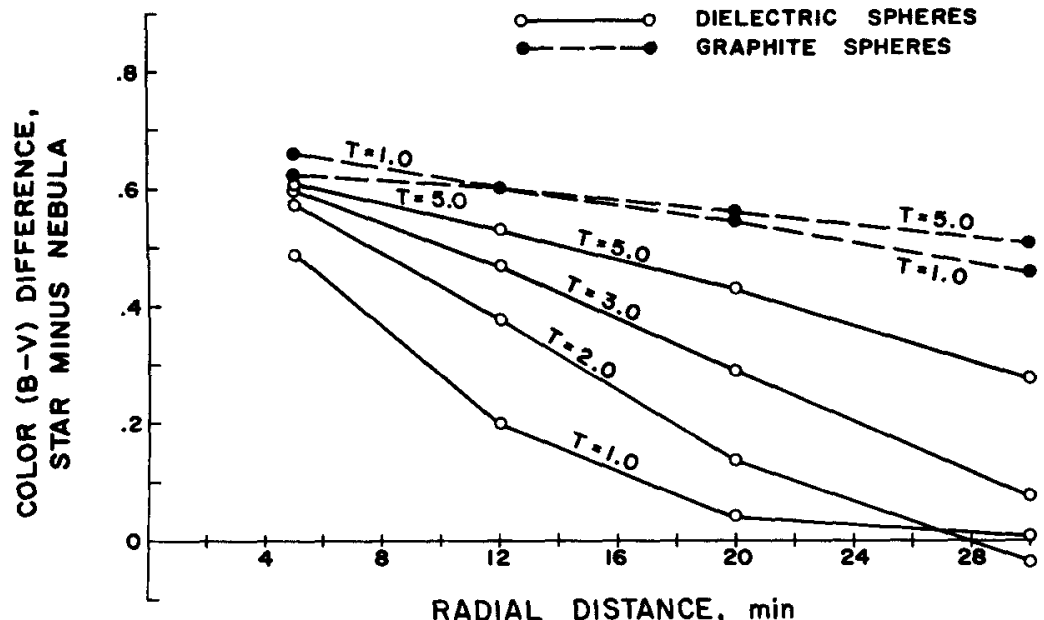

FIGURE 9.-Dependence of color difference on changes in model thickness $T$. Star behind; $a_{0}$ (dielectric spheres) $=0.50 \mu ; a$ (single size graphite spheres) $=0.05 \mu ; H=T+0.25$ pcs; $d=160 \mathrm{pcs} ; \beta=90^{\circ} ; \kappa(\lambda)_{V}=1.2$ magnitudes/pc.

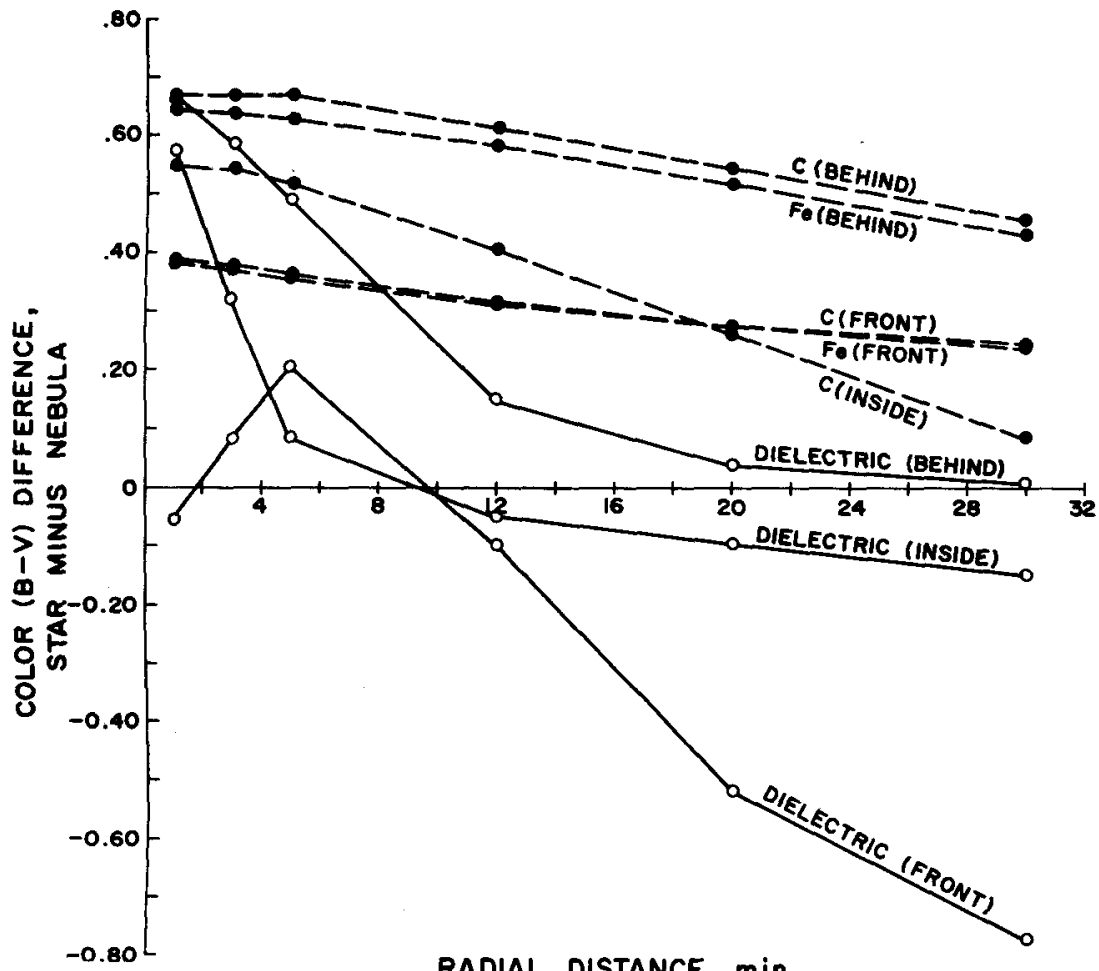

FIGURE 10. - Comparison of various geometrical models and particle compositions. $\beta=90^{\circ}$; $T=1.0 \mathrm{pc} ; d=160 \mathrm{pcs} ; \kappa(\lambda)_{V}=1.2$ magnitudes $/ \mathrm{pc} ; H$ (front) $=0.25 \mathrm{pc} ; H$ (inside) $=0.49$ pc; $H$ (behind) $=1.25$ pc. 
The spectral energy distribution for $\alpha$ Eri given in reference 7 was used for $I_{s}(\lambda)$. This is a B5 V star and thus is typical of those stars illuminating reflection nebulae. It was found that it made very little difference in the $B-V$ color differences whether a $\mathrm{B} 5 \mathrm{~V}$ or $\mathrm{A} 0 \mathrm{~V}$ star was used.

Three geometrical configurations are considered. Figure 1 illustrates the case of the star in front of the nebula. Results are also presented for the cases of star inside and star behind. In all the figures, the abscissa is in minutes of arc offset from the illuminating star.

Figure 2 is for the case of a star in front of the nebula with a tilt angle $\beta$ (see fig. 1) of $90^{\circ}$. The extreme sensitivity of the theory to the parameter $H$, the distance from the star to the front surface, is well shown here and in figures 7 and 8 . It can be seen that the predicted nebular colors may be either bluer or redder than the illuminating star or a mixture of both, depending on the value of $H$.

Figure 3 shows results for a model geometrically identical to that for figure 2 , but these data are for single size $(a=0.05 \mu)$ graphite particles. The curves illustrate the general qualitative behavior of graphite nebulae in that they have little slope or structure and do not go to red color difference values for star in front.

The model sensitivity to a change in nebular thickness $T$ is shown in figures 4 and 9 for star in front and star behind, respectively. The curves for $T=5$ parsecs are probably unrealistic within the framework of the approximate nature of our theory.

Figure 5 indicates the sensitivity of the model to the distance $d$, from the observer to the illuminating star. This parameter is often fairly well known and a small uncertainty in $d$ is of minor importance compared with the changes effected by a variation in $H$.

Figure 6 indicates the effects produced by variation of the linear extinction coefficient $\kappa(\lambda)$. It can be seen that by changing the tilt angle $\beta$ and $\kappa(\lambda)$, one may duplicate color difference curves rather closely. This fact is not surprising when one considers the large number of free parameters entering into the models.

Figure 10 is a composite diagram illustrating many of the changes in color differences due to overall geometry changes and due to scattering particle changes. Two curves for iron particles are also included.

The theoretical models of the present study indicate that the most fruitful observational approach would be to observe the nebula very close to the illuminating star and at moderate to large offset distances. These are the most difficult regions to measure: The first region is difficult because of the necessity of subtracting starlight scattered by the telescope optics, and the second region, because the nebular intensity is rapidly decreasing to less than that of the night sky. 


\section{REFERENCES}

1. Schalen, Carl: Studies of Reflection Nebulae. Uppsala Obs. Ann., vol. 1, no. 9, 1945.

2. Greenberc, J. M.: The Optics of Interstellar Grains. Proceedings of the I.A.U. Symposium No. 24: Spectral Classification and Multicolor Photometry (Saltsjobäden, Sweden), 1964.

3. OoRT, J. H.; and vaN DE Hulst, H. C.: Gas and Smoke in Interstellar Space. Bull. Astron. Inst. Netherlands, vol. 10, 1946, p. 187.

4. Whitrord, A. E.: An Extension of the Interstellar Absorption Curve. Astrophys. J., vol. 107, 1948, p. 102.

5. Hoyle, Fred; and Wickramasinghe, N. C.: On Graphite Particles as Interstellar Grains. Roy. Astron. Soc., Monthly Notices, vol. 124, 1962, p. 417.

6. VAN DE Hulst, H. C.: Light Scattering by Small Particles. John Wiley \& Sons, Inc., 1957.

7. Aller, L. H.; Faulkner, D. J.; and Norton, R. H.: Photoelectric Spectrophotometry of Selected Southern Stars. Astrophys. J., vol. 140, 1964, p. 1609.

\section{DISCUSSION}

Elvius: When you compare the color of the nebula with the color of the star, are you talking about the star as you actually see it?

Roark: We consider the fact that we are viewing the star through the nebulosity if it is behind or inside the front surface.

Elvius: Do you view the reddened star?

Roark: Yes. We tried to formulate this so that as few adjustments as possible to the observed data would have to be made.

Hall: Have you carried the computations very close to the star, within 2 or 3 seconds?

Roark: No, we have carried it only up to 1 minute offset.

Wickramasinghe: Have you used any graphite-core-ice-mantle grains in your model computations?

Roark: We are working on that. We have a program written but we're not satisfied with the results we are getting.

O'Dell: Have you treated this over a broader wavelength base than $U B V$ colors?

Roark: No.

O'Dell: The reason I ask is that any of these calculations, because of the shape of the size distribution, would be far more sensitive to a wider wavelength base; therefore, would it not be just as well to calculate color differences for an ultraviolet wavelength?

Roark: Yes. We plan to obtain $U-B$ color-difference models soon.

O'Dell: Could you indicate, at least in a qualitative manner, what the effects of second-order scattering would be? In the case of Merope the nebula is obviously optically thick, because the star count is very low when we look through the center of the nebula. I wonder if this is not something to worry about before one starts detailed interpretations? 
Roark: This is quite right.

Wickramasinghe: Concerning the expression for the size distribution you used: did you, use a size distribution for the graphite as well?

Roark: No, one size for graphite and iron, $0.05 \mu$, was used.

Wickramasinghe: What is the effect of changing size here?

Roark: There is very little structure to most of the metallic colordifference curves. Most are relatively flat, although some have a rather mild slope. If one uses your new refractive indices for graphite and a particle of $0.015-\mu$ radius, the color differences become about 0.4 bluer, but the shape of the curve does not change.

Wickramasinghe: $I$ see. The trouble with the graphite case is that you are essentially looking at scattering from different angles, and here you would really have to take into account the anisotropic properties of graphite.

Field: Is it fair to summarize qualitatively some of those graphs by saying that graphite always showed bluing, whereas the other particles did not always show bluing?

Roark: This is correct. We have many more graphs than we have shown and in no case yet can I get graphite or iron to go below the zero line. We cannot be sure why this occurs.

Wickramasinghe: In a qualitative treatment of reflection nebulae that Hoyle and I presented, we were able to get bluing as well as reddening for the nebula. We didn't use such a detailed model as yours, but we took into account the possibility of multiple scattering by Rayleigh scattering particles. The result was reddening for a large optical depth and bluing for a small optical depth.

Hall: Is there any combination for which the sort of reddening we observed in Merope would occur with only graphite particles.

Roark: Using our model and graphite parameters, we have not been able to get reddening, and we cannot obtain kinks in the color-difference curve.

Hall: Then these color data suggest, perhaps, that the nebular material is not graphite.

Roark: That is the conclusion that I am tempted to draw.

Elvius: Could anything be said about the albedo of these graphite particles? Can you compare the brightness of the nebula with that of the star in your models?

Roark: We have not looked into the problem of albedos as yet.

Elvius: I think the albedo of the graphite particles differs considerably from that of the dielectric particles. This might help in your interpretations.

Wickramasinghe: Did you do a calculation for a size distribution of graphite of the same sort as Oort-van de Hulst's?

Roark: I think in the early stages we did. but since then I had some 
reconsiderations of our theory and I would rather not quote the values. Our computation program is not complete. We want to try many size distributions which have been in the literature.

Wickramasinghe: It is important that computations for various size distributions of graphite be made before anything definite is concluded about the bluing or the reddening on the basis of this simple model. You might use different expressions for your ice-grain size distribution to see how sensitive your effect is going to be. It is not completely clear that the Oort-van de Hulst distribution which you have used is the correct one.

Roark: We used it because it was convenient, and it does give a good fit to the observed interstellar extinction law when used with dielectric spheres.

Wickramasinghe: For completeness, I think one ought to do other sorts of size distributions.

Greenberg: I agree, but in the present study, which is only a beginning, essentially two different models of grains were used; a single size graphite and a size distribution of the dielectric grains. Different models of clouds were studied within that framework.

Roark: One of the basic unknown parameters in this work is the linear extinction coefficient. I would like to have some independent means to get this.

Hall: Perhaps one could get some idea by studying the stars behind the nebulosity.

Strömgren: In that connection perhaps it is interesting to go back to Struve's work, in which rough estimates of the optical depths of reflection nebulae were made. I am sure that they are comparable to the Palomar Atlas, Dr. Hall.

Hall: Binnendijk has done this for Merope.

Strömgren: Well, Merope is a case where it appears big but even to an optical depth of 0.2 or so this would be reasonably within the range of your theory.

Roark: Yes, I think our theory would apply there. 